\title{
A SHRINKING COMPACT SYMMETRIC OBJECT: J11584+2450?
}

\author{
S. E. Tremblay, ${ }^{1}$ G. B. Taylor,${ }^{1,2}$ J. F. Helmboldt ${ }^{3}$ C. D. Fassnacht, ${ }^{4}$ and T. J. Pearson ${ }^{5}$ \\ Received 2007 December 5; accepted 2008 May 22
}

\begin{abstract}
We present multifrequency, multiepoch, Very Long Baseline Array (VLBA) observations of J11584+2450. These observations clearly show this source, previously classified as a core jet, to be a compact symmetric object (CSO). Comparisons between these new data and data taken over the last 11 years shows the edge-brightened hot spots retreating toward the core (and slightly to the west) at approximately $0.3 c$. Whether this motion is strictly apparent or actually physical in nature is discussed, as well as possible explanations, and what implications a physical contraction of J11584+2450 would have for current CSO models.
\end{abstract}

Subject headings: galaxies: active — galaxies: evolution — galaxies: individual (J11584+2450) — galaxies: jets — galaxies: nuclei — radio continuum: galaxies

\section{INTRODUCTION}

Compact symmetric objects (CSOs) are now a well-established class of radio sources loosely defined as sources with emission on both sides of the core (which itself is not always detected) on a size scale of $1 \mathrm{kpc}$ or less (Wilkinson et al. 1994). The generally accepted explanation for the small size of these objects is that they are young radio sources which could grow into larger FR II objects (Readhead et al. 1996; O’Dea 1998). Alternately, it has been proposed that the small size of these structures is due to their growth being frustrated by a dense environment (van Breugel et al. 1984; O'Dea 1998).

Due to their rapid growth, age estimates for the emission from these objects can be obtained kinematically (Owsianik \& Conway 1998; Taylor et al. 2000), yielding ages ranging from tens to thousands of years. Less accurate spectroscopic models (Readhead et al. 1996; Murgia et al. 1999; Gugliucci et al. 2005) place CSOs at a few thousands of years old. This generally supports the theory that these are young active galactic nuclei (AGNs) in the early stages of evolution. However, since the age distribution of currently known CSOs is heavily weighted on the younger side (Gugliucci et al. 2005), this indicates that the evolution might not be straightforward. This distribution should not be taken as definitive, however, since Gugliucci et al. concede it may be influenced by selection effects. One theory suggests that CSOs could be generally short-lived objects with only a small fraction of them surviving to become larger scale objects, while the remaining galaxies become permanently radio-quiet (Owsianik \& Conway 1998). In a competing theory there exists a cyclic process where unsuccessful CSOs have multiple opportunities to grow into larger objects (O’Dea \& Baum 1997). Alternatively, the current distribution could be an artifact of the small statistical sample from which it is derived combined with selection effects.

The picture presented in the above models might be overly simplistic. For example, all of these models predict continuous

\footnotetext{
1 Department of Physics and Astronomy, University of New Mexico, Albuquerque, NM 87131; tremblay@unm.edu, gbtaylor@unm.edu.

2 National Radio Astronomy Observatory, Socorro, NM 87801.

3 Naval Research Laboratory, Code 7213, Washington, DC 20375; joe .helmboldt@nrl.navy.mil.

4 Department of Physics, University of California, Davis, CA 95616; fassnacht@ solid.physics.ucdavis.edu.

5 Astronomy Department, California Institute of Technology, Pasadena, CA 91125; tjp@astro.caltech.edu.
}

radial expansion of the lobes, but CSOs such as 1031+567 (Taylor et al. 2000) have been observed with nonradial motion. Here we present observations of J11584+2450 (PKS 1155+251, SDSS $\mathrm{J} 115825.79+245018.0)$, a galaxy with a redshift of $0.20160 \pm$ 0.00040 (Zensus et al. 2002) that appears to be contracting toward its core on both sides.

Throughout this discussion, we assume $H_{0}=73 \mathrm{~km} \mathrm{~s}^{-1} \mathrm{Mpc}^{-1}$, $\Omega_{m}=0.27, \Omega_{\Lambda}=0.73$, so 1 mas $=3.213$ pc.

\section{OBSERVATIONS AND DATA REDUCTION}

Multifrequency observations of J11584+2450 were performed on 2006 September 19 with the Very Long Baseline Array (VLBA). A summary of these and other observations referred to in this paper is presented in Table 1 . These observations consisted of four $8 \mathrm{MHz}$ wide intermediate frequencies (IFs) in the $\mathrm{C}, \mathrm{X}$, and $\mathrm{U}$ bands with full polarization centered at 4605.5, 4675.5, 4990.5, 5091.5, 8106.0, 8176.0, 8491.0, 8590.0, 14902.5, 14910.5, 15356.5, and $15364.5 \mathrm{MHz}$ at an aggregate bit rate of $256 \mathrm{Mbps}$ to maximize $(u, v)$ coverage and sensitivity. When the data in each band were combined, the three central frequencies were 4844.7, 8344.7, and $15137.5 \mathrm{MHz}$. The integrations were performed in blocks ( $\sim 2$ minutes for 5 and $8 \mathrm{GHz}, \sim 7.5$ minutes for $15 \mathrm{GHz}$ ), and these blocks were spread out over a $9.5 \mathrm{hr}$ period to maximize $(u, v)$ coverage of the source.

Most of the calibration and initial imaging of the new data were carried out by automated AIPS (Greisen 2003) and DIFMAP (Shepherd 1997) scripts similar to those used in reducing the VIPS $5 \mathrm{GHz}$ survey data (Helmboldt et al. 2007; Taylor et al. 2005). To summarize, flagging of bad data and calibration were performed using the VLBA data calibration pipeline (Sjouwerman et al. 2005), while imaging was performed using DIFMAP scripts described in Taylor et al. (2005). Final imaging was performed manually using the DIFMAP program, with beam sizes of $1.906 \times$ 3.16 in position angle $-6.89^{\circ}, 1.195 \times 1.788$ in position angle $-3.58^{\circ}$, and $0.6876 \times 0.9794$ in position angle $-2.717^{\circ}$ for 5 , 8 , and $15 \mathrm{GHz}$, respectively.

\section{RESULTS \\ 3.1. Images}

Kellermann et al. (2004) observed J11584+2450 (B1155+251) as part of the VLBA $2 \mathrm{~cm}$ Survey. Since these observations were only at $15 \mathrm{GHz}$, they typically identified the brightest component 
TABLE 1

VLA AND VLBA ObServations of J1158+2450

\begin{tabular}{|c|c|c|c|c|c|c|c|}
\hline $\begin{array}{c}\text { Frequency } \\
(\mathrm{GHz})\end{array}$ & Date & $\begin{array}{c}\text { Time } \\
\text { (minutes) }\end{array}$ & $\begin{array}{c}\text { BW } \\
(\mathrm{MHz})\end{array}$ & Pol. & IFs & $\begin{array}{c}\text { Peak } \\
\left(\mathrm{mJy} \mathrm{beam}^{-1}\right)\end{array}$ & $\begin{array}{c}\mathrm{rms} \\
\left(\mathrm{mJy} \mathrm{beam}^{-1}\right)\end{array}$ \\
\hline $1.3649^{\mathrm{a}}$ & 2007 Mar 08 & 24.7 & 100 & 4 & 2 & 1073.7 & 0.1 \\
\hline $4.8447^{\mathrm{b}} \ldots$ & 2006 Sep 19 & 24.9 & 32 & 4 & 4 & 193.38 & 0.11 \\
\hline $8.3447^{\mathrm{b}} \ldots \ldots$ & 2006 Sep 19 & 26.9 & 32 & 4 & 4 & 118.38 & 0.17 \\
\hline $8.3541^{\mathrm{b}} \ldots \ldots \ldots \ldots \ldots \ldots \ldots \ldots \ldots \ldots \ldots \ldots \ldots$ & 2000 May 06 & 20.6 & 64 & 1 & 8 & 133.1 & 0.2 \\
\hline $15.138^{\mathrm{b}} \ldots \ldots \ldots \ldots \ldots \ldots \ldots \ldots$ & 2006 Sep 19 & 90.8 & 32 & 4 & 4 & 52.27 & 0.11 \\
\hline $15.335^{\mathrm{b}, \mathrm{c}} \ldots \ldots \ldots \ldots \ldots \ldots \ldots \ldots \ldots \ldots \ldots \ldots$ & 2001 Mar 04 & 57.0 & 56 & 1 & 1 & 74.85 & 0.31 \\
\hline $15.335^{\mathrm{b}, \mathrm{c}} \ldots \ldots \ldots \ldots \ldots \ldots \ldots$ & 1999 May 21 & 37.6 & 56 & 1 & 1 & 82.73 & 0.33 \\
\hline $15.350^{\mathrm{b}, \mathrm{c}} \ldots \ldots \ldots \ldots \ldots \ldots \ldots \ldots \ldots \ldots \ldots$ & 1995 Apr 07 & 44.9 & 32 & 1 & 1 & 110.0 & 0.3 \\
\hline
\end{tabular}

a VLA observation.

b VLBA observation.

c These data were taken as part of the VLBA $2 \mathrm{~cm}$ Survey (Kellermann et al. 2004).

in an image to be the core, and consequently classified this source as a core jet with the core being the southern, bright component.

Figure 1 shows the 5, 8, and $15 \mathrm{GHz}$ VLBA images made from the 2006 September observations of J11584+2450. The $15 \mathrm{GHz}$ map shows the clearest structure, and so is used to label components of the source. The $15 \mathrm{GHz}$ image shows a compact unresolved component $(\mathrm{C})$ with resolved emission both to the north (N1) and the south (S1). The southern emission then seems to have another component that expands out toward the west (W2). There also exists some weak emission on the western edge of the image ( W1; with a peak flux density of $\left.0.55 \mathrm{mJy} \mathrm{beam}^{-1}\right) . \mathrm{S} 1$ is the brightest component in the image ( $52.3 \mathrm{mJy} \mathrm{beam}^{-1}$ peak), and is what was previously identified as the core. In the $8 \mathrm{GHz}$ image the edges of $\mathrm{C}, \mathrm{N} 1, \mathrm{~S} 1$, and $\mathrm{W} 2$ become indistinguishable, but these components can still be identified by local peaks within the image. Interestingly, an eastern spur develops from the southern edge of N1, extending in the opposite direction from the majority of the diffuse emission. Overall the emission appears more extended, and $\mathrm{W} 1$ has a more significant detection. The $5 \mathrm{GHz}$ map further smears the interior components together until only $\mathrm{N} 1$ and S1 are clearly visible as local maxima of the map. The spur mentioned above becomes brighter, and the western emission stretches out farther toward a very well detected W1 (3.83 mJy beam ${ }^{-1}$ peak flux density). There is emission from the eastern spur toward the south in this image (hereafter the $5 \mathrm{GHz}$ southeastern clump), which has an integrated flux density of $3.78 \mathrm{mJy}$.

The geometry between N1, C, and S1 was measured using the $200615 \mathrm{GHz}$ data, since those components are most distinguishable. The axial ratio, $\mathrm{N} 1 / \mathrm{S} 1=1.59$, and the angle subtended between the arms, N1-C-S1, is $166.9^{\circ}$.

We used the VLA in the D configuration to investigate what appeared to be an extension of the western emission to kiloparsec scale from the NRAO VLA Sky Survey (NVSS; Condon et al. 1998), but found no indication of any western emission from $\mathrm{J} 11584+2450$ (Fig. 2) and the previous extension to be a result of the higher rms $\left(0.45 \mathrm{mJy}\right.$ beam $\left.^{-1}\right)$ of the NVSS compared to our image with rms $=0.085 \mathrm{mJy}^{\text {beam }}{ }^{-1}$.

In addition, we acquired a visual image (Fig. 3) from the Sloan Digital Sky Survey Data Release 5 (SDSS DR5; AdelmanMcCarthy et al. 2007). This shows the source to have a galaxy $4^{\prime \prime}$ to the southeast, which is uncataloged outside of the SDSS. Two different algorithms have been used to determine a multicolor photometric redshift for this object from the SDSS image. The first algorithm utilized the template fitting method and yields $z=0.0008 \pm 0.0226$ (Csabai et al. 2003), while the second algorithm used a neural network method and yields $z=0.065 \pm$
0.123 (Oyaizu et al. 2008), placing an upper limit of $z=0.19$, which is comparable to J11584+2450's redshift.

\subsection{Spectral Index Distribution}

The 2006 VLBA images at 8 and $15 \mathrm{GHz}$ were matched in resolution in order to obtain a spectral index distribution across the source that was overlaid onto a $5 \mathrm{GHz}$ image to show overall source structure (Fig. 4). This distribution clearly shows a compact flat-spectrum component $\left(\alpha \approx-0.276\right.$, where $\left.F_{\nu} \propto \nu^{\alpha}\right)$ situated between two steeper spectrum lobes $(\alpha \approx-1.03)$. More steep-spectrum emission is found to the west of these lobes $(\alpha \approx$ -1.36 to -1.54$)$, where it then fades below the detection threshold at high frequencies. Looking at the $5 \mathrm{GHz}$ southeastern clump and using its peak, the spectral index would have to be steeper than -4.35 for the emission to fall below the rms of the $8 \mathrm{GHz}$ image. Alternatively, the absence of this feature could be due to having fewer short spacings at $8 \mathrm{GHz}$, or it could merely be an imaging artifact at $5 \mathrm{GHz}$.

\section{DISCUSSION}

\subsection{Reclassification of J11584+2450}

The compact flat-spectrum component seen in Figure 4 is compatible with emission from the nucleus, or core, of a galaxy (Begelman et al. 1984). The steeper spectrum components extending north and south from the core are accordant with the spectral signature of jets or hot spots. This overall structure is clearly consistent with $\mathrm{J} 11584+2450$ being a CSO.

\subsection{Component Motions}

To characterize intrinsic motions within the source, a multicomponent elliptical Gaussian model was made of the 1999 data and then applied to the 1995, 2001, and $200615 \mathrm{GHz}$ data, varying the flux and position parameters of each Gaussian component (Table 2). The extended dual-lobed structure in the 1995 data (but notably missing from the 1999, 2001, and 2006 images) of what is now considered the core was modeled using a single component, since it is likely this extension is only an artifact.

Each of the four IFs of the 2006 data was then individually modeled and compared to each other to determine the systematic error $\left(\sigma_{\text {sys }}\right)$ associated with modeling each component. The total position error $\left(\sigma_{\text {tot }}\right)$ was then calculated for individual components using $\sigma_{\text {tot }}^{2}=\sigma_{\text {stat }}^{2}+\sigma_{\text {sys }}^{2}$, where $\sigma_{\text {stat }}$ is the expected statistical error associated with modeling Gaussian components in twodimensional polar coordinates (Table 3; adapted from onedimensional case per Fomalont [1999]). Since all model positions 

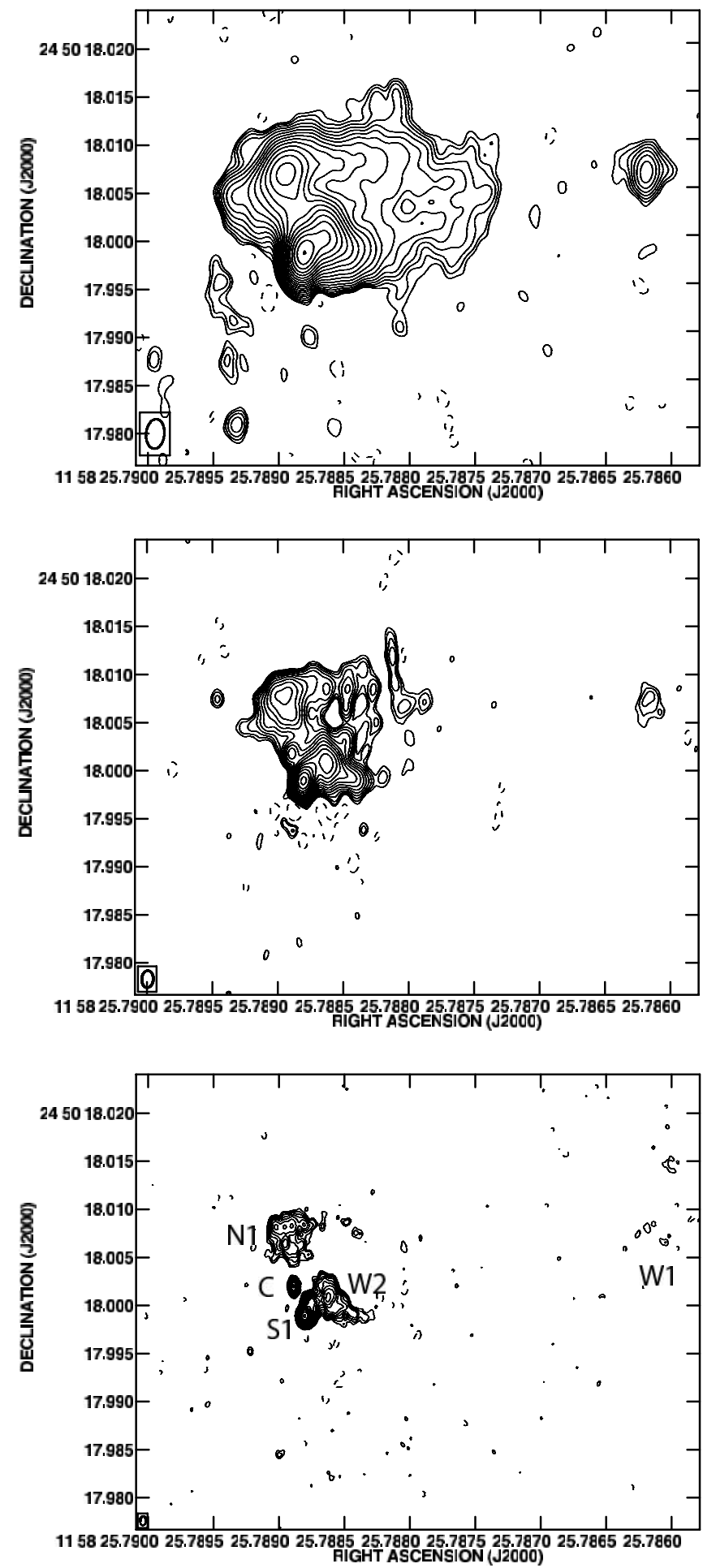

FIG. 1.-VLBA observations from 2006 September of J11584+2450 at frequencies of ( from top to bottom) 4.84, 8.34, and $15.13 \mathrm{GHz}$. Contour levels begin at $0.375 \mathrm{mJy}_{\text {beam }}{ }^{-1}$ and increase by factors of $2^{1 / 2}$.

are referenced to $\mathrm{C}, \sigma_{\text {sys }}$ for the core is accounted for by the other component uncertainties. Since the calibrated data sets from the VLBA $2 \mathrm{~cm}$ Survey are each averaged to one frequency, the $\sigma_{\text {sys }}$ attained from the 2006 data was applied to them as well. Similarly, a multicomponent Gaussian model was made of the 2000 data and then applied to the $20068 \mathrm{GHz}$ data, varying the flux and position parameters of each Gaussian component (Table 4). The $\sigma_{\text {sys }}$ values were obtained for each component using the four

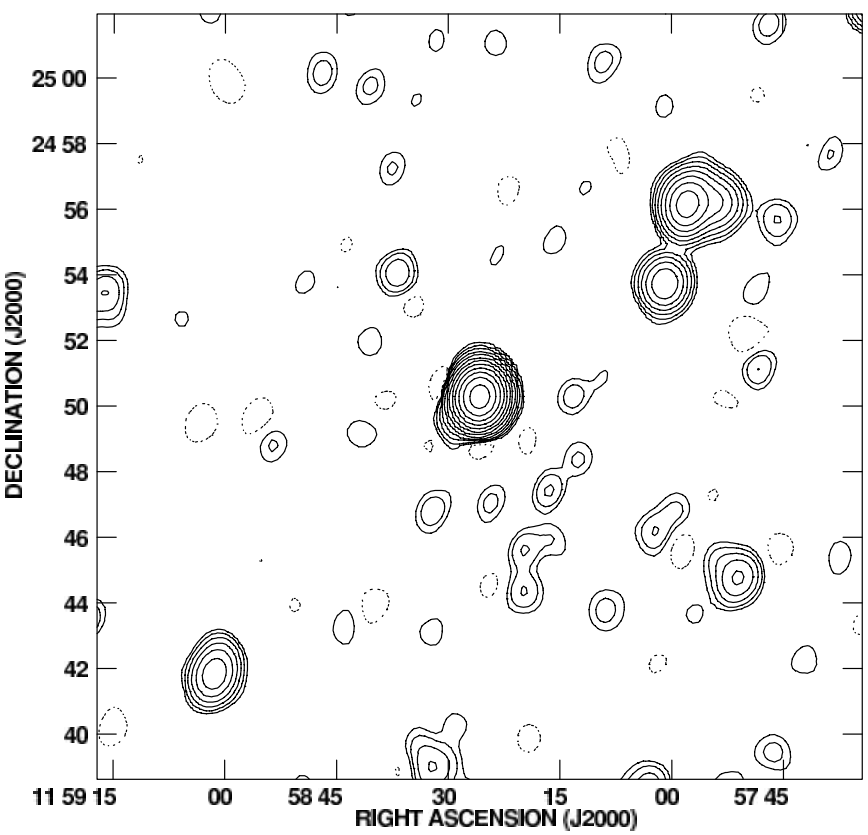

FIG. 2.-VLA observations from 2007 March centered on J11584+2450 at $1.3649 \mathrm{GHz}$ in D configuration. The contour levels begin at $0.336 \mathrm{mJy}^{\text {beam }^{-1}}$ and increase by levels of 2 . The source shows no significant structure at this frequency and resolution, but does show possible extension to the southeast.

IFs as above in the 2006 data, and using the four IFs closest to those same frequencies in the 2000 data (Table 5).

These models were used to calculate component velocities relative to the core for each band, which are plotted in Figure 5 with the tail of each vector located at the earliest data position in the band. The components representing the hot spots at the working surface of the jets which were modeled (N1 and S1) appear to have contracted toward the core as well as traveled westward, and this motion is consistent between the two bands. In addition,

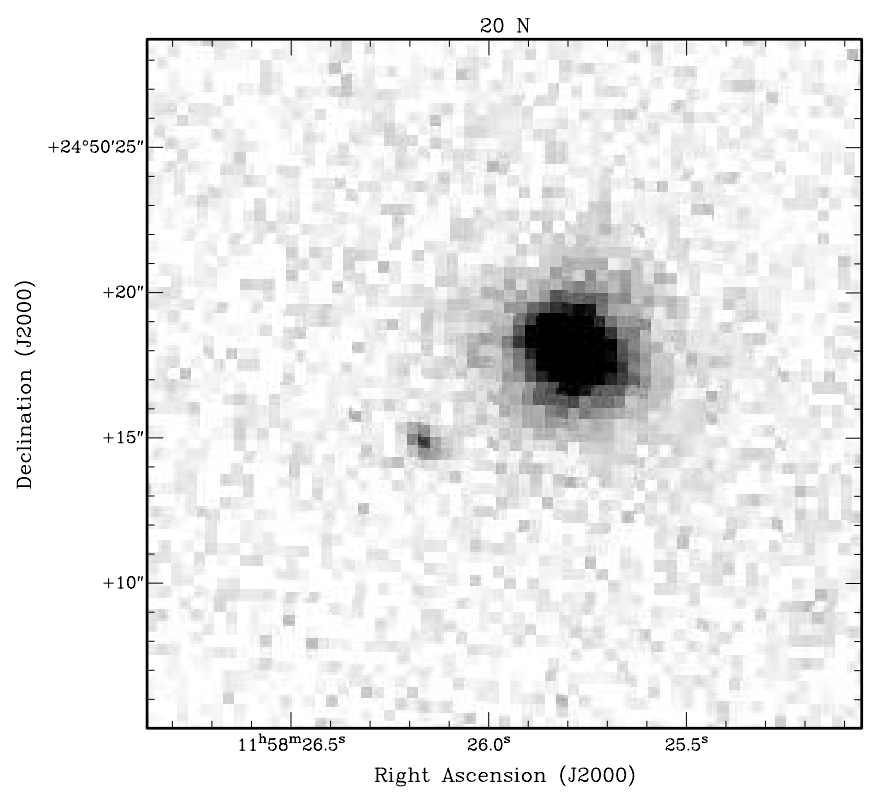

FIG. 3.- This $R$-band SDSS image of J11584+2450 also shows a second source 4 " to the southeast (SDSS J115826.16+245014.9). If these sources have the same redshift, then there is $13 \mathrm{kpc}$ separation between them. In this image, the magnitude of J11854+2450 is $17.68 \pm 0.01$, while the magnitude of SDSS $\mathrm{J} 115826.16+245014.9$ is $21.99 \pm 0.06$. 


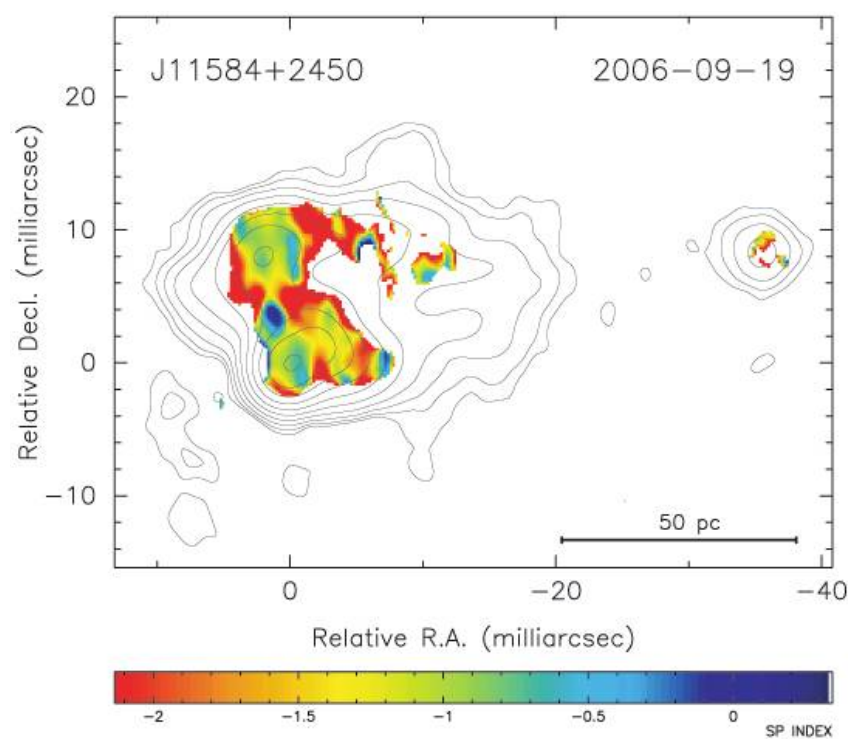

Fig. 4.- - Multifrequency observations of a newly identified CSO (J11584+ 2450); $5 \mathrm{GHz}$ contours overlaid on an $8-15 \mathrm{GHz}$ spectral index image. Note the flat-spectrum core, as well as the symmetric dual-lobed structure in the source. Also, the emission abruptly bends to the west. This sudden path change, and the steep-spectrum compact knot at the western edge, are not clearly understood.

the W2 component moves toward the northwest in both bands. Performing a least-squares fit to solve for the velocities in each band separately, and then using these independent values to reduce the error, yields a radial contraction velocity (normalized to the speed of light) of $0.42 \pm 0.03 c$ for S1 and $0.20 \pm 0.07 c$ for N1. Overlaying the contour maps of different epochs (see Fig. 6 for one example) is also supportive of contraction, since the 1995 contours are interior to the 2006 contours, suggesting that the component motion is not an artifact of the modeling.

This motion was not detected by the VLBA $2 \mathrm{~cm}$ Survey over the $6 \mathrm{yr}$ interval between 1995 and 2001, since the velocities

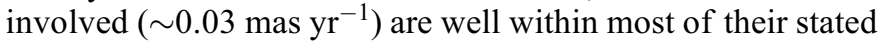

velocity errors for this source (Kellermann et al. 2004). The total flux density of the source has been decreasing steadily since at least 1995; the models show that this drop can be attributed to S1 decreasing steadily ( $40 \%$ decrease at $15 \mathrm{GHz}$ over this $11 \mathrm{yr}$ period), while the other components exhibit small fluctuations.

\subsection{Apparent Motion Interpretation}

Since actual contraction of the source toward the core is something that has not been previously observed, we first examine the reasons behind an effect that would merely cause apparent motion in the system. One possible explanation for seeing the contraction of this source is that if hot spots are advancing out away from the core and expanding and younger hot spots are brightening due to interactions at the end of the jet, then the models might not be fit to the same components. The largest problems with this hypothesis are its lack of explanation of both the western emission and the western component to the hot spot velocities, which means these properties require a separate, unrelated explanation if the contraction is to be explained by hot spot dimming and advance.

\subsection{Physical Motion Interpretations}

Leaving open the possibility that the data represent physical motions in the system, we include discussion along those lines. One interpretation is that we are viewing a projection effect caused by rotation of the source. While solid-body rotation is an unphysical scenario, it gives us an idea about what fluid rotation would look like for this system, so we consider it as a first approximation of rotational motion. The angular velocity of the rotation is dependent on the initial orientation. Assuming the axis of rotation lies in the plane of the sky, and the inclination angle is less than $45^{\circ}$, since larger values would yield Doppler boosting, the jets would have a rotational period between just 260 and $1880 \mathrm{yr}$.

Since the system is a fluid and not a rigid body, it would actually have differential rotation. The core would therefore be spinning

TABLE 2

$15 \mathrm{GHz}$ Gaussian Model Components

\begin{tabular}{|c|c|c|c|c|c|c|c|c|c|}
\hline Component & Epoch & $\underset{(\mathrm{Jy})}{S}$ & $\begin{array}{c}r \\
\text { (mas) }\end{array}$ & $\begin{array}{c}\sigma_{r} \\
(\mathrm{mas})\end{array}$ & $\begin{array}{c}\theta \\
(\mathrm{deg})\end{array}$ & $\begin{array}{c}\sigma_{\theta} \\
(\mathrm{deg})\end{array}$ & $\begin{array}{c}a \\
\text { (mas) }\end{array}$ & $b / a$ & $\begin{array}{c}\Phi \\
(\operatorname{deg})\end{array}$ \\
\hline \multirow[t]{4}{*}{$\mathrm{C}$} & 1995 & 0.0167 & 0.000 & 0.007 & 0.00 & 0.00 & 0.283 & 1.00 & -18.57 \\
\hline & 1999 & 0.0331 & 0.000 & 0.003 & 0.00 & 0.00 & 0.283 & 1.00 & -18.57 \\
\hline & 2001 & 0.0170 & 0.000 & 0.007 & 0.00 & 0.00 & 0.283 & 1.00 & -18.57 \\
\hline & 2006 & 0.0120 & 0.000 & 0.003 & 0.00 & 0.00 & 0.283 & 1.00 & -18.57 \\
\hline \multirow[t]{4}{*}{$\mathrm{S} 1$} & 1995 & 0.1267 & 3.647 & 0.014 & -161.28 & 0.15 & 0.272 & 0.86 & 50.59 \\
\hline & 1999 & 0.0945 & 3.514 & 0.014 & -159.79 & 0.15 & 0.272 & 0.86 & 50.59 \\
\hline & 2001 & 0.0798 & 3.400 & 0.014 & -159.03 & 0.15 & 0.272 & 0.86 & 50.59 \\
\hline & 2006 & 0.0765 & 3.360 & 0.014 & -160.12 & 0.15 & 0.272 & 0.86 & 50.59 \\
\hline \multirow[t]{4}{*}{$\mathrm{N} 1$.............................. } & 1995 & 0.0327 & 5.610 & 0.052 & 9.55 & 1.12 & 3.02 & 0.345 & 29.62 \\
\hline & 1999 & 0.0288 & 5.801 & 0.049 & 8.37 & 0.95 & 3.02 & 0.345 & 29.62 \\
\hline & 2001 & 0.0257 & 5.497 & 0.045 & 9.52 & 1.78 & 3.02 & 0.345 & 29.62 \\
\hline & 2006 & 0.0399 & 5.325 & 0.038 & 6.82 & 0.50 & 3.02 & 0.345 & 29.62 \\
\hline \multirow[t]{4}{*}{$\mathrm{W} 2 \ldots \ldots \ldots \ldots$} & 1995 & 0.0637 & 3.226 & 0.034 & -127.77 & 0.46 & 2.72 & 0.32 & -81.22 \\
\hline & 1999 & 0.0541 & 3.775 & 0.033 & -115.06 & 0.46 & 2.72 & 0.32 & -81.22 \\
\hline & 2001 & 0.0369 & 3.746 & 0.033 & -115.65 & 2.65 & 2.72 & 0.32 & -81.22 \\
\hline & 2006 & 0.0630 & 3.435 & 0.030 & -116.83 & 0.34 & 2.72 & 0.32 & -81.22 \\
\hline
\end{tabular}

Notes.-Parameters of each Gaussian component of the model brightness distribution are as follows: Component, Gaussian component; Epoch, year of observation (see Table 1 ); $S$, flux density; $r, \sigma_{r}, \theta, \sigma_{\theta}$, polar coordinates (and the associated errors) of the center of the component relative to the center of component $\mathrm{C}$; $a$, semimajor axis; $b / a$, axial ratio; and $\Phi$, component orientation. All angles are measured from north through east. 
TABLE 3

15 GHz Gaussian Model Components from Selected IFs

\begin{tabular}{|c|c|c|c|c|c|c|c|c|c|c|c|}
\hline Component & Epoch & $\begin{array}{c}r_{1} \\
\text { (mas) }\end{array}$ & $\begin{array}{c}r_{2} \\
\text { (mas) }\end{array}$ & $\begin{array}{c}r_{3} \\
\text { (mas) }\end{array}$ & $\begin{array}{c}r_{4} \\
\text { (mas) }\end{array}$ & $\begin{array}{l}\sigma_{r(\mathrm{sys})} \\
(\mathrm{mas})\end{array}$ & $\begin{array}{c}\theta_{1} \\
(\mathrm{deg})\end{array}$ & $\begin{array}{c}\theta_{2} \\
\text { (deg) }\end{array}$ & $\begin{array}{c}\theta_{3} i \\
(\mathrm{deg})\end{array}$ & $\begin{array}{c}\theta_{4} \\
\text { (deg) }\end{array}$ & $\begin{array}{c}\sigma_{\theta(\mathrm{sys})} \\
(\mathrm{deg})\end{array}$ \\
\hline $\mathrm{S} 1$ & 2006 & 3.368 & 3.353 & 3.341 & 3.377 & 0.014 & -159.93 & -160.10 & -160.36 & -160.13 & 0.15 \\
\hline 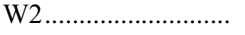 & 2006 & 3.473 & 3.430 & 3.390 & 3.440 & 0.030 & -116.45 & -116.63 & -117.06 & -117.30 & 0.34 \\
\hline
\end{tabular}

NoтEs.-Parameters of each Gaussian component of the IF model position distribution are as follows: Component, Gaussian component; Epoch, year of observation (see Table 1); $r_{1}-r_{4}$, radial positions of model components in IFs 1 through 4 , respectively; $\sigma_{r \text { (sys) }}$, the standard deviation in radial position; $\theta_{1}-\theta_{4}$, the polar angular position of model components in IFs 1 through 4 , respectively; and $\sigma_{\theta(\mathrm{sys})}$, the standard deviation in polar angular position. All angles are measured from north through east.

TABLE 4

$8 \mathrm{GHz}$ Gaussian Model Components

\begin{tabular}{|c|c|c|c|c|c|c|c|c|c|}
\hline Component & Epoch & $\begin{array}{c}S \\
\text { (Jy) }\end{array}$ & $\begin{array}{c}r \\
(\mathrm{mas})\end{array}$ & $\begin{array}{c}\sigma_{r} \\
(\mathrm{mas})\end{array}$ & $\begin{array}{c}\theta \\
(\mathrm{deg})\end{array}$ & $\begin{array}{c}\sigma_{\theta} \\
(\mathrm{deg})\end{array}$ & $\begin{array}{c}a \\
\text { (mas) }\end{array}$ & $b / a$ & $\begin{array}{c}\Phi \\
(\mathrm{deg})\end{array}$ \\
\hline \multirow[t]{2}{*}{$\mathrm{C}$} & 2000 & 0.0273 & 0.000 & 0.001 & 0.00 & 0.00 & 0.214 & 1.00 & 63.74 \\
\hline & 2006 & 0.0115 & 0.000 & 0.003 & 0.00 & 0.00 & 0.214 & 1.00 & 63.74 \\
\hline \multirow[t]{2}{*}{$\mathrm{S} 1$} & 2000 & 0.1581 & 3.240 & 0.017 & -157.29 & 0.15 & 0.4875 & 0.71 & -23.38 \\
\hline & 2006 & 0.1507 & 3.082 & 0.022 & -155.45 & 0.19 & 0.4875 & 0.71 & -23.38 \\
\hline \multirow[t]{2}{*}{$\mathrm{N} 1$} & 2000 & 0.1005 & 5.597 & 0.055 & 6.75 & 0.31 & 2.195 & 0.76 & -38.51 \\
\hline & 2006 & 0.1098 & 5.508 & 0.049 & 5.27 & 0.10 & 2.195 & 0.76 & -38.51 \\
\hline \multirow[t]{2}{*}{ W2 . } & 2000 & 0.1505 & 3.653 & 0.028 & -109.43 & 0.32 & 1.57 & 0.80 & 40.08 \\
\hline & 2006 & 0.1489 & 3.703 & 0.011 & -105.95 & 0.47 & 1.57 & 0.80 & 40.08 \\
\hline
\end{tabular}

Notes.-Parameters of each Gaussian component of the model brightness distribution are as follows: Component, Gaussian component; Epoch, year of observation (see Table 1); $S$, flux density; $r, \sigma_{r}, \theta, \sigma_{\theta}$, polar coordinates (and the associated errors) of the center of the component relative to the center of component $\mathrm{C}$; $a$, semimajor axis; $b / a$, axial ratio; and $\Phi$, component orientation. All angles are measured from north through east.

TABLE 5

$8 \mathrm{GHz}$ Gaussian Model Components from Selected IFs

\begin{tabular}{|c|c|c|c|c|c|c|c|c|c|c|c|}
\hline Component & Epoch & $\begin{array}{c}r_{1} \\
\text { (mas) }\end{array}$ & $\begin{array}{c}r_{2} \\
\text { (mas) }\end{array}$ & $\begin{array}{c}r_{3} \\
\text { (mas) }\end{array}$ & $\begin{array}{c}r_{4} \\
\text { (mas) }\end{array}$ & $\begin{array}{l}\sigma_{r(\mathrm{sys})} \\
(\mathrm{mas})\end{array}$ & $\begin{array}{c}\theta_{1} \\
(\mathrm{deg})\end{array}$ & $\begin{array}{c}\theta_{2} \\
(\mathrm{deg})\end{array}$ & $\begin{array}{c}\theta_{3} i \\
(\operatorname{deg})\end{array}$ & $\begin{array}{c}\theta_{4} \\
(\mathrm{deg})\end{array}$ & $\begin{array}{l}\sigma_{\theta \text { (sys) }} \\
(\mathrm{deg})\end{array}$ \\
\hline $\mathrm{S} 1$ & 2000 & 3.225 & 3.270 & 3.250 & 3.237 & 0.017 & -157.64 & -157.48 & -157.26 & -157.29 & 0.15 \\
\hline \multirow[t]{2}{*}{$\mathrm{N} 1 \ldots \ldots \ldots \ldots \ldots \ldots \ldots \ldots \ldots$} & 2000 & 5.573 & 5.675 & 5.535 & 5.551 & 0.055 & 7.31 & 6.65 & 7.05 & 6.54 & 0.31 \\
\hline & 2006 & 5.467 & 5.501 & 5.586 & 5.467 & 0.049 & 5.17 & 5.34 & 5.19 & 5.39 & 0.09 \\
\hline 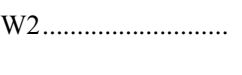 & 2000 & 3.607 & 3.673 & 3.675 & 3.642 & 0.028 & -109.24 & -109.98 & -110.07 & -109.45 & 0.32 \\
\hline
\end{tabular}

Notes.-Parameters of each Gaussian component of the IF model position distribution are as follows: Component, Gaussian component; Epoch, year of observation (see Table 1); $r_{1}-r_{4}$, radial positions of model components in IFs 1 through 4 , respectively; $\sigma_{r \text { (sys) }}$, the standard deviation in radial position; $\theta_{1}-\theta_{4}$, the polar angular position of model components in IFs 1 through 4, respectively; and $\sigma_{\theta(\mathrm{sys})}$, the standard deviation in polar angular position. All angles are measured from north through east. 


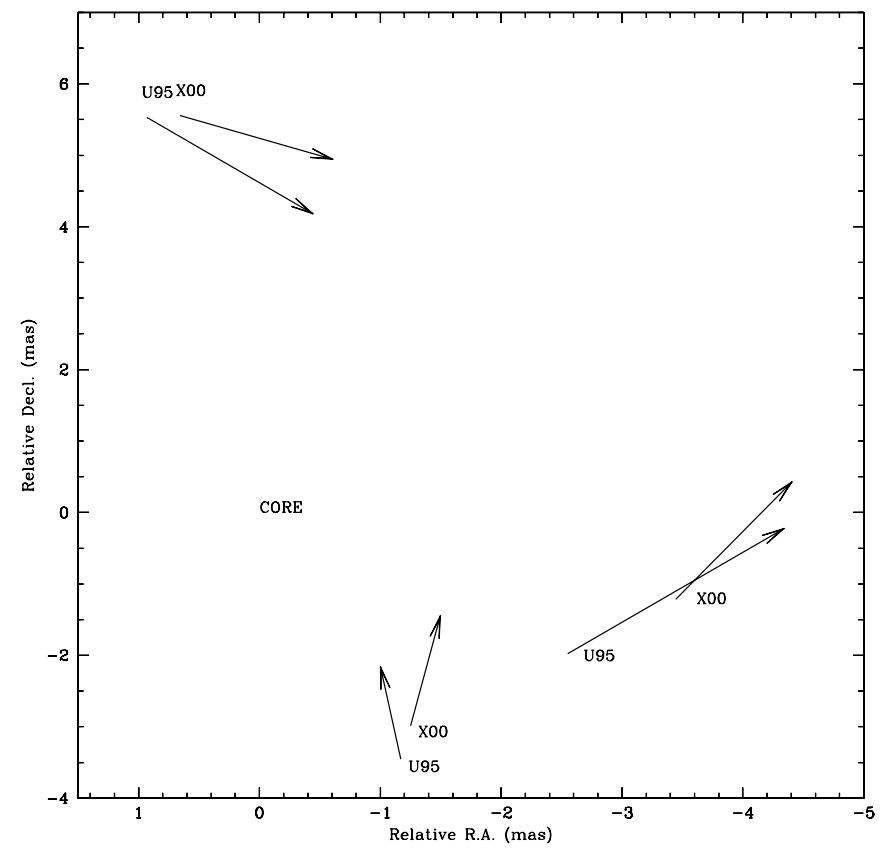

FIG. 5.-Relative velocity of model components. Velocity of each Gaussian model component is plotted $(1$ mas $=0.2 c)$ with the tail of each vector originating at the model component's position at its earliest observation ( 1995 for $15 \mathrm{GHz}$ and 2000 for $8 \mathrm{GHz}$ ). The model fits are in agreement with the contour overlay plots in showing this source to be shrinking.

even faster than the observed jet components, and we would expect the core to appear highly variable, since the base of the jet would frequently be pointed toward us. A second physical interpretation of the motion is precession. The westward component to the velocities of both jets strongly argues against precession. If the jets were precessing and thus appearing shorter, they would move in opposite directions (i.e., one moves east, while the other moves west) as well as inward. In addition, these hypotheses fail to explain the older western emission.

Another physical elucidation of the motion is that there exists some reason for the pressure of the environment to increase, then this could leave the jets underpressured and lead to contraction of $\mathrm{J} 11584+2450$. Such a pressure change could result from a relative motion between a clumpy environment and the CSO. The $1.59 \mathrm{~N} 1 / \mathrm{S} 1$ axial ratio is also indicative of the jets encountering a dense environment. If the departure of the ratio from 1 was due to Doppler boosting, then the brighter hot spot would be farther from the core ( $\mathrm{S} 1$ in the case of $\mathrm{J} 11584+2450$ ). This would yield a ratio smaller than 1; therefore, the ratio is likely due to the jet running into difficulty as it tunnels through the environment, causing it to be both shorter and brighter. Both the angle N1-C-S1 and the western deviation of the emission are consistent with the source moving eastward and being influenced by ram pressure similar to what has been observed in wide-angle tailed radio sources (e.g., 3C 465; Hardcastle et al. 2005; Sakelliou \& Merrifield 2000), but on a smaller spatial scale. Relative motion could exist between $\mathrm{J} 11584+2450$ and its host galaxy, causing the interstellar medium to produce ram pressure against the radio jet. If the companion galaxy to the southeast (Fig. 3) is at a similar redshift and these two galaxies are members of a cluster, then $\mathrm{J} 11584+2450$ might be moving toward the center of the gravitational potential. However, this is highly speculative, and more observations are needed to determine the cluster environment.

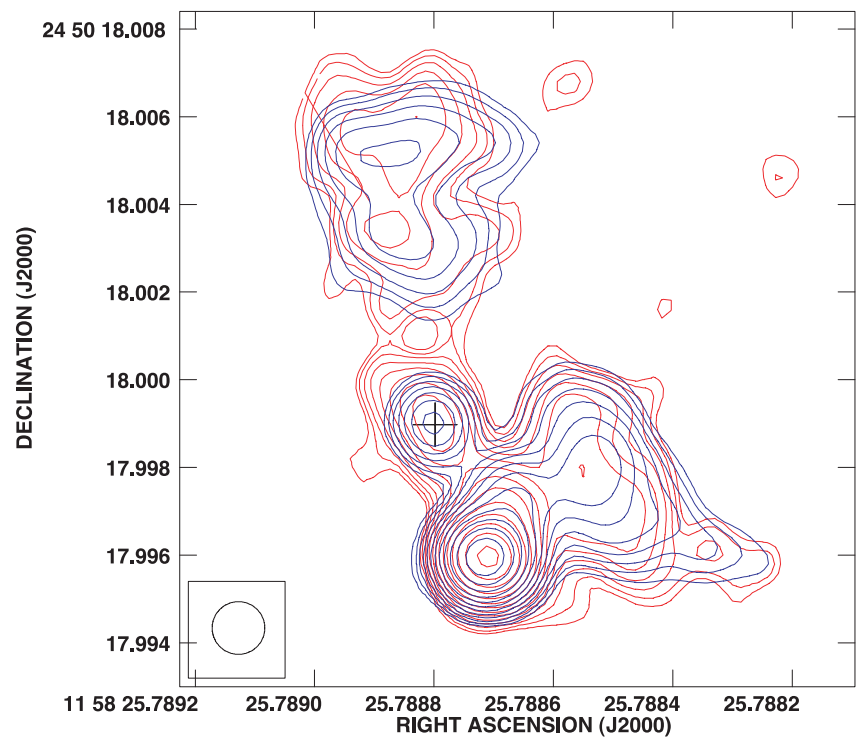

FIG. 6.- Two-epoch overlay of J11584+2450; $15 \mathrm{GHz}$ data from 1995 (red contours) are plotted with the $15 \mathrm{GHz}$ data from 2006 (blue contours). This figure shows contraction of the source toward the core (cross) over time. The contour levels begin at $1.10 \mathrm{mJy}^{\text {beam }}{ }^{-1}$ and increase by factors of $2^{1 / 2}$.

\section{CONCLUSIONS}

After analyzing multifrequency $(5,8$, and $15 \mathrm{GHz})$ VLBA data from radio source $\mathrm{J} 11584+2450$ we reclassify it as a CSO. Fitting the data with multicomponent Gaussian models and overlaying images of different epochs on each other not only shows that this source is not growing at the usual rate of $\sim 0.1 c-0.3 c$, but also that each jet is apparently shrinking in size at $\sim 0.3 c$ and each is additionally moving westward at $\sim 0.2 c$. Confirmation of other CSOs having either recessive behavior or nonradial motion like 1031+567 (Taylor et al. 2000) would mean the current models need to be modified to allow for possible nonlinear growth periods during the evolution of AGNs. The prospect of nonlinear growth for CSOs would further bring into question the validity of kinematic ages. Gugliucci et al. (2005) found seven out of the 13 CSOs they dated to be under $500 \mathrm{yr}$ old and commented that the expectation for a steady state population of CSOs would have a uniform distribution of ages. While this is a small statistical sample, it is also what one would expect to see if CSOs spend a greater fraction of time as small sources. However, in kinematic observations of $\sim 10$ CSOs we have seen contraction in just one source.

Future VLBA observations of this source are planned to follow the motion of the components and to see whether they continue to recede toward the core, and over what timescale this occurs. Lower frequency $(1.4 \mathrm{GHz})$ observations should be carried out to confirm the existence of the $5 \mathrm{GHz}$ southeastern clump. If the emission from both jets is actually flowing toward the west, then lower frequency observations might also show W1 merging with the diffuse western emission, and could reveal larger scale structures.

We thank an anonymous referee for constructive suggestions. The National Radio Astronomy Observatory is a facility of the National Science Foundation operated under cooperative agreement by Associated Universities, Inc.

Facilities: VLA, VLBA 
Adelman-McCarthy, J. K., et al. 2007, ApJS, 172, 634

Begelman, M. C., Blandford, R. D., \& Rees, M. J. 1984, Rev. Mod. Phys., 56, 255

Condon, J. J., Cotton, W. D., Greisen, E. W., Yin, Q. F., Perley, R. A., Taylor, G. B., \& Broderick, J. J. 1998, AJ, 115, 1693

Csabai, I., et al. 2003, AJ, 125, 580

Fomalont, E. B. 1999, in ASP Conf. Ser. 180, Synthesis Imaging in Radio Astronomy II, ed. G. B. Taylor, C. L. Carilli, \& R. A. Perley (San Francisco: ASP), 301

Greisen, E. W. 2003, in Information Handling in Astronomy: Historical Vistas, ed. A. Heck (Dordrecht: Kluwer), 109

Gugliucci, N. E., Taylor, G. B., Peck, A. B., \& Giroletti, M. 2005, ApJ, 622, 136

Hardcastle, M. J., Sakelliou, I., \& Worrall, D. M. 2005, MNRAS, 359, 1007

Helmboldt, J. F., et al. 2007, ApJ, 658, 203

Kellermann, K. I., et al. 2004, ApJ, 609, 539

Murgia, M., Fanti, C., Fanti, R., Gregorini, L., Klein, U., Mack, K.-H., \& Vigotti, M. 1999, A\&A, 345, 769

O'Dea, C. P. 1998, PASP, 110, 493

O’Dea, C. P., \& Baum, S. A. 1997, AJ, 113, 148
REFERENCES

Owsianik, I., \& Conway, J. E. 1998, A\&A, 337, 69

Oyaizu, H., Lima, M., Cunha, C. E., Lin, H., Frieman, J., \& Sheldon, E. S. 2008, ApJ, 674, 768

Readhead, A. C. S., Taylor, G. B., Xu, W., Pearson, T. J., Wilkinson, P. N., \& Polatidis, A. G. 1996, ApJ, 460, 612

Sakelliou, I., \& Merrifield, M. R. 2000, MNRAS, 311, 649

Shepherd, M. C. 1997, in ASP Conf. Ser. 125, Astronomical Data Analysis Software and Systems VI, ed. G. Hunt \& H. E. Payne (San Francisco: ASP), 77

Sjouwerman, L. O., Mioduszewski, A. J., \& Greisen, E. W. 2005, in ASP Conf. Ser. 340, Future Directions in High Resolution Astronomy, ed. J. Romney \& M. Reid (San Francisco: ASP), 613

Taylor, G. B., Marr, J. M., Pearson, T. J., \& Readhead, A. C. S. 2000, ApJ, 541, 112

Taylor, G. B., et al. 2005, ApJS, 159, 27

van Breugel, W., Miley, G., \& Heckman, T. 1984, AJ, 89, 5

Wilkinson, P. N., Polatidis, A. G., Readhead, A. C. S., Xu, W., \& Pearson, T. J. 1994, ApJ, 432, L87

Zensus, J. A., Ros, E., Kellermann, K. I., Cohen, M. H., Vermeulen, R. C., \& Kadler, M. 2002, AJ, 124, 662 